\title{
Interrupción voluntaria del embarazo en Latinoamérica, superando barreras
}

\section{Voluntary interruption of the pregnancy in Latin America, overcoming barriers}

\author{
Adriana I González-Q ${ }^{1,2} \mathbb{D}^{\mathbb{D}}$; Diva J Moreno-L ${ }^{3}{ }^{\mathbb{D}}$; David E Grass- $\mathrm{C}^{3} \mathbb{D}^{\mathbb{D}}$
}

Forma de citar: González A, Moreno D, Grass DE. Interrupción voluntaria del embarazo en Latinoamérica, superando barreras. Salud UIS. 53: e21013. doi: https://doi.org/10.18273/saluduis.53.e:21013 @ @)

\begin{abstract}
Resumen
Introducción: el embarazo no planeado o no intencional y el aborto son situaciones que afectan la vida de mujeres a nivel mundial, sin distinción de etnia, edad, riqueza, o ubicación geográfica, sin embargo, tiene una mayor posibilidad de presentarse y generar consecuencias negativas en mujeres con ciertas determinantes sociales. Objetivo: mostrar el estado actual de la interrupción voluntaria del embarazo en países de Latinoamérica y del Caribe con énfasis en el reciente avance de la legislación argentina sucedido durante diciembre del 2020. Metodología: se realizó una búsqueda no estructurada de información sobre la legislación del aborto en países de Latinoamérica y del Caribe y se hizo una revisión de tema sobre aspectos actuales y relevantes de la interrupción voluntaria del embarazo. Conclusiones: es necesario que prestadores de servicios de salud y sociedad latinoamericana repasen las lecciones aprendidas de diferentes países sobre las consecuencias negativas para la salud de las mujeres y sus familias debido a las restricciones para acceder al aborto seguro. El mejoramiento de la calidad y las capacidades de los sistemas de salud en los países de bajos y medianos recursos, con mayor inversión e investigación en temas de salud sexual y reproductiva, resultará en la eliminación de barreras e inequidades en la prestación de atención médica a las mujeres, respetando sus derechos y autonomía.
\end{abstract}

Palabras clave: Aborto; Aborto Legal; Aborto Inducido; Aborto Criminal; Aborto Terapéutico; Misoprostol; Educación Sexual; Telemedicina.

\begin{abstract}
Introduction: Unplanned or unintended pregnancy and abortion are situations that affect the lives of women worldwide without distinction of ethnicity, age, economic level, or geographical location. However, they have a greater probability of occurring and negative consequences in women with certain social determinants. Objective: Our main objective is to show the current state of the Voluntary Interruption of Pregnancy in Latin American and Caribbean countries with special emphasis on the recent advance of the legislation of Argentina that occurred last

1. Universidad Industrial de Santander. Bucaramanga, Colombia.

2. Universidad de Santander UDES. Bucaramanga, Colombia.

3. Hospital local. Cabrera, Santander, Colombia.

Correspondencia: Adriana González. Dirección: Calle 54 \# 49-09. Altos de Pan de Azúcar. Bucaramanga. Teléfono: +57 3162337387. Correo electrónico: adrigonz@correo.uis.edu.co
\end{abstract}


December. Methodology: An unstructured search for information about Abortion Legislation in Latin American and Caribbean countries was carried out and a subject revision on current and relevant aspects of Voluntary Interruption of Pregnancy was made. Conclusions: It is necessary that as Health Service providers and as a Latin American Society, we review the lessons learned from different countries about the negative consequences on the health of women and their families due to the restrictions for accessing legal abortions. Improving the quality and capacity of the health system in low- and middle- income countries, in addition to greater investment and research in sexual and reproductive health issues, will derive a removal of barriers and inequity related to the provision of medical attention for women while respecting their rights and autonomy.

Keywords: Abortion; Legal Abortion; Abortion Induced; Abortion Criminal; Therapeutic Abortion; Misoprostol; Sex Education; Telemedicine.

\section{Introducción}

El embarazo no planeado o no intencional y el aborto son situaciones que afectan la vida de mujeres a nivel mundial sin distinción de etnia, edad, nivel de riqueza o ubicación geográfica. Sin embargo, tiene una mayor probabilidad de presentación y afectación en mujeres con ciertas determinantes sociales ${ }^{1,2}$. Por ejemplo, en Estados Unidos las mujeres negras tienen una tasa de aborto 3,4 veces mayor que las mujeres blancas y las mujeres hispanas tienen 1,7 veces mayor probabilidad de aborto que las mujeres blancas ${ }^{3}$. Esto refleja una marcada disparidad por etnia ${ }^{1}$.

Los países con mayores restricciones al aborto también enfrentan tasas sustancialmente más altas de embarazos no planeados. A esta clase de embarazos le anteceden problemáticas como: una deficiente educación sexual integral, la falta de programas de planificación familiar y el difícil acceso a servicios esenciales de salud reproductiva en comparación con otros países.

De acuerdo con los datos del Instituto GuttmacherLancet y la Organización mundial de la Salud (OMS), entre 2015 y 2019, ocurrieron en el mundo aproximadamente 121 millones de embarazos no planeados por año, correspondiente a una tasa global de 64 por 1000 mujeres en edades de 15 a 49 años. Esto demuestra una tendencia hacia la disminución, si se compara con las tasas de los 25 años anteriores, que alcanzaron un promedio de 79 por 1000 mujeres de 15 a 49 años $^{1}$. En algunas regiones o comunidades ${ }^{3}$ se ve reflejado un mayor acceso a servicios de planificación familiar y a anticonceptivos modernos.

Del total de embarazos no planeados, aproximadamente $61 \%$ terminaron en aborto (73 millones/año), correspondiente a una tasa global de 39 por 1000 mujeres en edades entre 15 y 49 años. De estos abortos, un $45 \%$ se llevaron a cabo en un ambiente inseguro, que dio origen a un $7,9 \%$ de las muertes maternas ${ }^{3}$.
La tasa mundial de aborto ha permanecido estable desde hace 30 años, sin embargo, se evidenció una leve tendencia hacia la disminución en los años 20002005. Aunque en el último quinquenio (2015-2019) se presentó una nueva elevación, no se observa que sea menor en países o regiones donde el aborto está restringido $^{1}$. En países desarrollados donde el aborto está totalmente despenalizado hay cifras bajas de embarazo no intencionado y de aborto, mientras que en los países de bajos y medianos recursos, en donde por lo general hay restricción parcial o total del aborto, la proporción de embarazo no deseado y finalización de este por medio de aborto es mayor.

Esto muestra que los progresos globales no han sido uniformes y que el gran esfuerzo de organizaciones mundiales expertas en salud sexual y reproductiva (SSR) no ha sido suficiente. Persisten las complicaciones del aborto inseguro en países de medianos y bajos recursos, principalmente, en aquellos donde el aborto no ha sido despenalizado ${ }^{1}$. En los países que tienen amplias restricciones para el ejercicio de un aborto seguro o interrupción voluntaria del embarazo (IVE), las mujeres se ven obligadas a realizar un aborto en condiciones no seguras con alta morbilidad y consecuencias deletéreas para su salud física y mental, incluso con probabilidad de morir cuando estas muertes son prevenibles ${ }^{4}$.

Los valores religiosos, tendencias políticas y la interpretación moral de las instancias involucradas no son una situación menor, ya que en un momento dado podrían facilitar u obstaculizar el acceso a anticonceptivos modernos y a servicios seguros de IVE. Incluso, en países donde la legislación del aborto no es totalmente restrictiva, es decir que está despenalizado en ciertas causales, las mujeres siguen teniendo barreras para ejercer su autonomía en temas de SSR y para acceder a servicios de aborto seguro ${ }^{5}$. 


\section{IVE en tiempo de pandemia}

En el actual contexto de emergencia sanitaria ocasionado por la pandemia de COVID-19, y con las medidas de aislamiento preventivo y confinamiento, las barreras de acceso a servicios de SSR, entre ellos los programas de anticoncepción y la IVE, se han acentuado y han originado efectos negativos que afectan la salud y la vida de las mujeres, jóvenes y niñas, especialmente de las más vulnerables ${ }^{6}$. Desde el mes de marzo del año 2020, la OMS instó a los Estados a que se respetara y garantizara la disponibilidad y acceso a los servicios de SSR como servicios de salud esenciales, alertando que su restricción constituye una violación a los derechos humanos ${ }^{7}$. Adicionalmente, el 28 de Abril del 2020, el Fondo de Población de las Naciones Unidas (UNFPA) estimó que la violencia basada en género podría aumentar en un $20 \%$ durante los períodos de confinamiento obligatorio, incluyendo los casos de violencia sexual, lo que, sumado al limitado acceso a la planificación familiar, indudablemente aumentaría los embarazos no deseados ${ }^{8}$.

Durante el mismo mes, el Grupo de Trabajo Interagencial sobre Salud Reproductiva en Situaciones de Crisis emite la orientación programática para la salud sexual y reproductiva en contextos humanitarios y frágiles durante la pandemia por el COVID-19 y recomiendan asegurar que se brinde apoyo para la autoadministración del aborto inducido con medicamentos hasta las 12 semanas de gestación; a fin de mejorar el acceso a la atención, se puede evaluar la posibilidad de utilizar sistemas remotos para ofrecer orientación en la autoadministración?.

En Colombia, el 30 de marzo del 2020, el Ministerio de Protección Social expidió la Resolución 536 de $2020^{10}$, por la cual se dictó el "plan de acción para la prestación de servicios de salud durante las etapas de contención y mitigación de la pandemia por SARSCoV-2 (covid-19)"; en ella se restringe la prestación de algunos de los procedimientos contemplados en la Ruta de Atención en Salud Materno Perinatal ${ }^{11}$, exceptuando el suministro de métodos anticonceptivos y la atención de la IVE en los términos establecidos en la Sentencia C-355 de 2006, sin suspender la asesoría y prestación del servicio anticonceptiva posevento y otros servicios de salud que se deben seguir garantizando ${ }^{10}$.

\section{IVE en Colombia}

Con más de 10 años previos de debate legal y social, finalmente, la Corte Constitucional colombiana reconoció en mayo de 2006, con la sentencia C-355 de 2006, que el aborto o interrupción voluntaria del embarazo es un derecho íntimamente ligado al derecho, a la vida, salud, integridad, autodeterminación, intimidad y dignidad de las mujeres; y se despenalizó en tres causales: (1.) cuando peligra la vida o la salud de la madre (entendiendo la salud como el estado de bienestar físico, mental y psicosocial); (2.) cuando se presenta malformación del feto incompatible con la vida; (3.) cuando el embarazo es producto de abuso, violación, incesto, transferencia de óvulo o inseminación no consentida ${ }^{12}$.

El Ministerio de Salud y Protección Social, como ente regulador del Sistema de Seguridad Social en Salud, y de acuerdo con la jurisprudencia constitucional, rige las acciones para asegurar que todas las mujeres en el territorio nacional tengan un libre y efectivo ejercicio de sus derechos sexuales y reproductivos; por esto, emiten, con la asesoría del UNFPA y la OMS, los documentos guía para la prevención del aborto inseguro en Colombia ${ }^{13,14}$ y los documentos técnicos de apoyo para los prestadores de servicios de salud, en cuanto a la atención del aborto seguro, incluyendo el primer nivel de atención ${ }^{15-17}$, la asesoría en anticoncepción posaborto ${ }^{18}$ y la guía de capacitación para los prestadores de la atención ${ }^{19}$.

\section{Legislación del aborto}

En el año 2017, el Center for Reproductive Rights agrupó los países, a nivel mundial, en 5 categorías (I-V), teniendo en cuenta los motivos por los cuales es válido el aborto en cada uno de ellos (Figura 1), ubicando dentro de América Latina y el Caribe (ALC) a 8 países de los 26 del mundo que se hallan en la categoría I; estos tienen leyes que prohíben de forma total el aborto, es decir que no está permitido bajo ninguna circunstancia. Ellos son: Aruba, Curazao, República Dominicana, El Salvador, Haití, Honduras, Jamaica y Nicaragua. Cabe anotar que Haití está llevando a cabo la actualización de su código penal, donde se incluye la propuesta de avanzar en la despenalización del aborto ${ }^{46}$ (véase Tabla 1). 


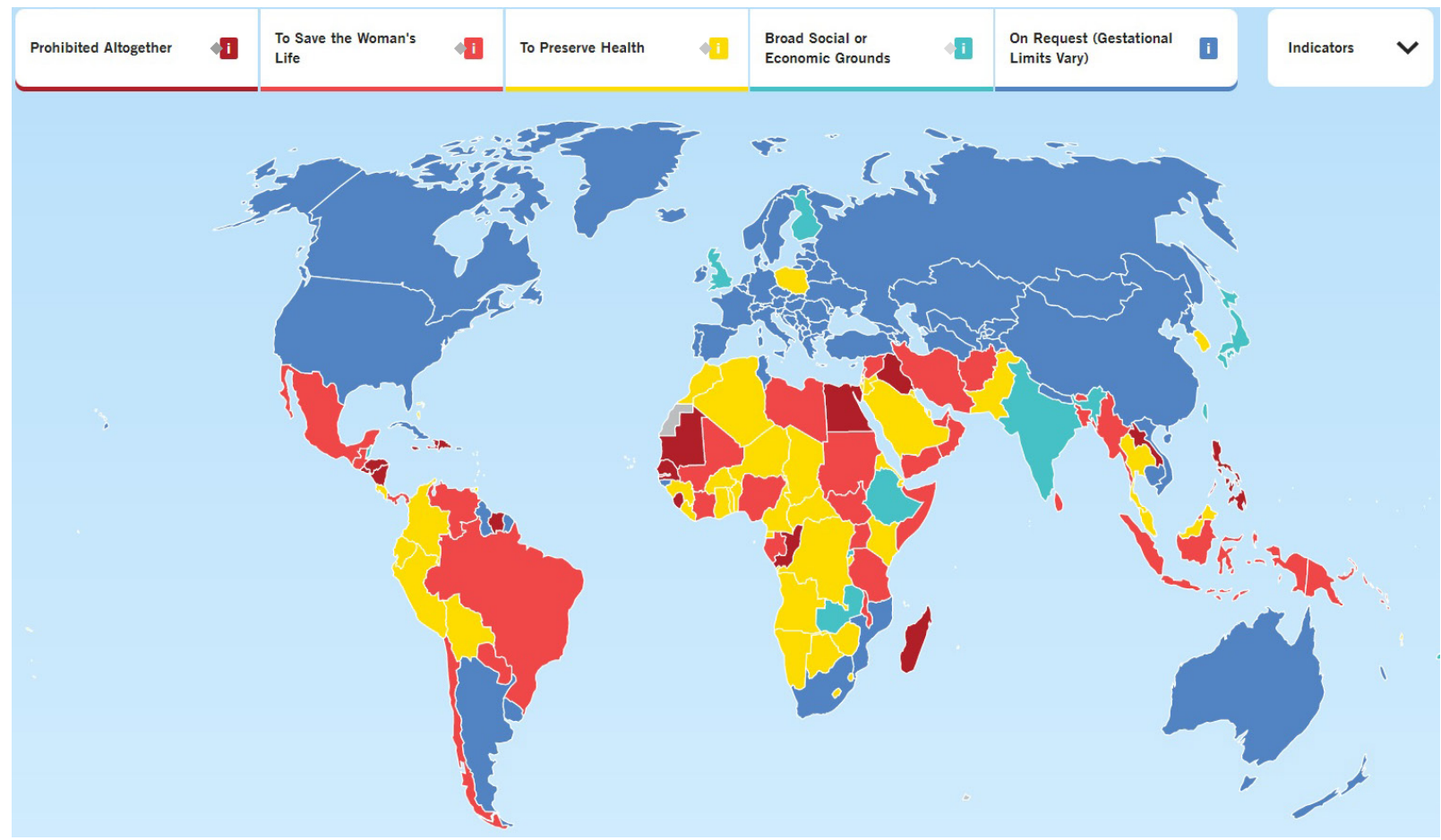

Figura 1. Mapa mundial sobre el estado legal del aborto en el mundo. Tomado de: https://reproductiverights.org/worldabortionlaws (Autorizada su publicación por Center Anticonception Rights.)

Tabla 1. Basada en el mapa mundial del estado legal del aborto en el mundo, en: https://reproductiverights.org/ worldabortionlaws? country=BRA (diseño: David Grass).

\begin{tabular}{|c|c|c|c|}
\hline País & Año & Título guía o legislación & Limite EG \\
\hline Haití & 1835 & Código penal de Haití. Art. $262^{20}$ & Penalizado en todas las causales \\
\hline $\begin{array}{l}\text { República } \\
\text { Dominicana }\end{array}$ & 1884 & Código penal de la República Dominicana. Art. $317^{21}$ & Penalizado en todas las causales \\
\hline El Salvador & 1997 & $\begin{array}{l}\text { Código penal del salvador; "De los delitos relativos a la } \\
\text { vida del ser humano en formación." Art. 133-137 }\end{array}$ & Penalizado en todas las causales \\
\hline Honduras & 2017 & $\begin{array}{c}\text { Código penal de Honduras, Capitulo II; Aborto. Art. } \\
\text { 126-132 } \\
\text { Código penal de Honduras Decreto no. 130-2017, } \\
\text { Aborto. Art. } 196^{24}\end{array}$ & Penalizado en todas las causales \\
\hline Nicaragua & 2007 & $\begin{array}{l}\text { Código penal de la república de Nicaragua. Capitulo II, } \\
\text { Art. } 143-145^{25}\end{array}$ & Penalizado en todas las causales \\
\hline $\begin{array}{l}\text { México (excepto } \\
\text { Ciudad de México y } \\
\text { Oaxaca) }\end{array}$ & 1931 & Código penal Federal. Capítulo VI, Art. 329-334 26 & $\begin{array}{l}\text { Sin límite: Violación o delito sexual, } \\
\text { Riesgo para la vida de la mujer }\end{array}$ \\
\hline Guatemala & 1973 & $\begin{array}{c}\text { Código Penal de Guatemala, Decreto No. 17-73, Titulo } \\
\text { VII, Capítulo III, Art. 133-140 }\end{array}$ & $\begin{array}{c}\text { Sin límite: Riesgo para la vida de la } \\
\text { mujer }\end{array}$ \\
\hline Panamá & 2007 & $\begin{array}{c}\text { Texto único del código penal de la República de } \\
\text { Panamá, Capítulo 1, Sección 3; Aborto provocado, Art. } \\
141-144^{28}\end{array}$ & $\begin{array}{l}\text { Sin límite: Riesgo para la vida de la } \\
\text { madre, violación o delito sexual }\end{array}$ \\
\hline Venezuela & 2000 & $\begin{array}{l}\text { Código Penal de Venezuela, Título VIII, Capítulo IV; } \\
\text { Del aborto provocado, Art. } 432-436^{29 n u}\end{array}$ & $\begin{array}{c}\text { Sin límite: Riesgo para la vida de la } \\
\text { mujer }\end{array}$ \\
\hline Brasil & 1940 & $\begin{array}{l}\text { Decreto-Ley N. } .^{\circ} 2.848 \text {, de } 07.12 .1940 \text {, alterado pela } \\
\text { Ley N. } 9.777 \text { em 26/12/98. Código Penal Brasil, Parte } \\
\text { Especial, Título I, Capítulo I, Art. 124- } 128^{30}\end{array}$ & $\begin{array}{l}\text { Sin límite: Riesgo para la vida de } \\
\text { la mujer, Violación o delito sexual, } \\
\text { Malformaciones fetales }\end{array}$ \\
\hline
\end{tabular}




\begin{tabular}{|c|c|c|c|}
\hline País & Año & Título guía o legislación & Limite EG \\
\hline Paraguay & 1997 & $\begin{array}{l}\text { Código penal de la república del Paraguay. Ley No. } \\
\text { 1160/97, Art. 349-353 }\end{array}$ & $\begin{array}{c}\text { Sin límite: Riesgo para la vida de la } \\
\text { madre }\end{array}$ \\
\hline Chile & 2018 & $\begin{array}{c}\text { Acompañamiento y atención integral a la mujer que se } \\
\text { encuentra en alguna de las tres causales que regula la } \\
\text { ley } 21.030^{32}\end{array}$ & $\begin{array}{c}\text { En caso de violación: } \\
12 \text { semanas }>14 \text { años } \\
14 \text { semanas }<14 \text { años } \\
20 \text { semanas; } 1 \text { causal; mujeres que se } \\
\text { encuentran en riesgo vital } \\
\text { Sin límite: patología congénita } \\
\text { adquirida, riesgo para la vida de la } \\
\text { mujer }\end{array}$ \\
\hline Costa Rica & 1970 & $\begin{array}{l}\text { Código Penal de Costa Rica, Ley N. }{ }^{\circ} 4573 \text {,Art. 118-122 } \\
\text { y } 381^{33}\end{array}$ & $\begin{array}{c}\text { Sin límite: Riesgo para la salud de la } \\
\text { mujer }\end{array}$ \\
\hline Trinidad y Tobago & 1967 & Abortion Act 1967 of United Kingdom. Chapter $87^{34}$ & $\begin{array}{l}24 \text { semanas: Riesgo para la salud } \\
\text { física o mental de la mujer o de } \\
\text { cualquier miembro de la familia } \\
\text { Sin límite: Riesgo para la vida de la } \\
\text { mujer, malformaciones fetales }\end{array}$ \\
\hline Colombia & 2006 & Sentencia 355 del $2006^{12}$ & $\begin{array}{l}\text { Sin límite: Violación o delito sexual, } \\
\text { riesgo para la salud de la mujer, } \\
\text { malformación fetal }\end{array}$ \\
\hline Ecuador & 2014 & Código orgánico integral penal, Art. $147-150^{35}$ & $\begin{array}{c}\text { Sin límite: Riesgo para la salud de la } \\
\text { mujer }\end{array}$ \\
\hline Perú & 1991 & $\begin{array}{l}\text { Código Penal, decreto legislativo N. }{ }^{\circ} \text {, Capitulo II, Art. } \\
\qquad 114-120^{36}\end{array}$ & $\begin{array}{c}\text { Sin límite: Riesgo para la salud de la } \\
\text { mujer }\end{array}$ \\
\hline Bolivia & 1972 & $\begin{array}{l}\text { Código Penal de Bolivia, Titulo VIII, Capitulo II, } \\
\text { Artículos } 263-269^{37}\end{array}$ & $\begin{array}{l}\text { Sin límite: Riesgo para la salud de } \\
\text { la madre, violación o delito sexual, } \\
\text { incesto }\end{array}$ \\
\hline Belice & 2005 & $\begin{array}{l}\text { Belize criminal code chapter } 101 \text {, Title IX, } \\
\text { Art. } 111,112,127^{38}\end{array}$ & $\begin{array}{l}\text { Sin límite: Por motivos } \\
\text { socioeconómicos, riesgo para la salud } \\
\text { de la madre, malformaciones fetales }\end{array}$ \\
\hline $\begin{array}{l}\text { Estados de México: } \\
\text { Ciudad de México } \\
\text { (DF) y Oaxaca }\end{array}$ & 2002 & $\begin{array}{l}\text { Código Penal Ciudad de México (2002), } \\
\text { Artículos } 144-150^{39}\end{array}$ & $\begin{array}{l}12 \text { semanas: A petición de la mujer } \\
\text { Sin límite: Riesgo para la salud de } \\
\text { la mujer, violación o delito sexual, } \\
\text { malformaciones fetales }\end{array}$ \\
\hline Cuba & 1965 & $\begin{array}{l}\text { En cuba no existe una ley de aborto pero se encuentra } \\
\text { despenalizado desde } 1965^{40,41} \\
\text { Se penaliza el aborto ilegal en: Código penal, Capítulo } \\
\text { IV Art. } 267-271^{42}\end{array}$ & 12 semanas: A petición de la mujer \\
\hline Guyana francesa & 1975 & Article L.2212-1 du Code de la santé publique ${ }^{43}$ & $\begin{array}{l}12 \text { semanas por voluntad de la mujer } \\
\text { Sin límite: por alguna razón médica }\end{array}$ \\
\hline Argentina & 2020 & $\begin{array}{l}\text { Ley interrupción voluntaria del embarazo } \\
\qquad 30 \text { diciembre } 2020^{44}\end{array}$ & $\begin{array}{l}14 \text { semana: A petición de la mujer } \\
\text { Sin límite: riesgo para la madre o } \\
\text { malformación del feto }\end{array}$ \\
\hline Uruguay & 2016 & Sentencia N. ${ }^{\circ} 586 / 2015^{45}$ & $\begin{array}{l}12 \text { semanas: A petición de la mujer } \\
14 \text { semanas por violación } \\
\text { Sin límite: riesgo para la madre o } \\
\text { malformación del feto }\end{array}$ \\
\hline
\end{tabular}

En la categoría II, cuando las leyes permiten el aborto para salvar la vida de la mujer, en ALC se encuentran 13 de 39 países a nivel mundial: Antigua y Barbuda, Brasil, Chile, Dominicana, Guatemala, México (sin los estados Distrito Federal y Oaxaca), Panamá, Paraguay y Venezuela.
En la categoría III, con leyes para preservar la salud de la mujer, en ALC se encuentran 10 de los 56 países a nivel global, entre ellos: Bahamas, Bolivia, Colombia, Costa Rica, Ecuador, Perú, Sait Kitts and Nevis, Santa Lucía, Granada, Trinidad y Tobago. 
En la Categoría IV se encuentran 3 de los 14 países que a nivel mundial tienen leyes que permiten el aborto por amplias razones. Estas leyes, por lo general, se interpretan liberalmente para permitir el aborto en una amplia gama de circunstancias como el entorno real o razonablemente previsible de una mujer y sus circunstancias sociales o económicas. En esta categoría se encuentra Barbados, Belice, Saint Vicent and the Granadies.

En la categoría V, donde el aborto es legal en todas las condiciones y con límites de edad gestacional, en ALC se encuentran Cuba, Puerto Rico, Guyana, Uruguay, los estados mexicanos Distrito Federal de México y Oaxaca y, recientemente, Argentina.

\section{Historia de una lucha de las mujeres en Argentina}

La lucha por los derechos sexuales y reproductivos, impulsada desde grupos feministas y por el movimiento de mujeres, inicia en Argentina la Campaña Nacional por el Derecho al Aborto Legal Seguro y Gratuito, lanzada el 28 de mayo de 2005, con adhesión de más de 500 organizaciones de todo el país. El objetivo era instalar en la sociedad y en el Estado el debate en torno a la necesidad de despenalizar y legalizar el aborto en Argentina, lo cual contribuyó a que más mujeres y organizaciones se sumaran al proceso y lograran la aprobación de una norma legal ${ }^{47}$.

Entre sus acciones, la campaña elaboró el Proyecto de Ley de Interrupción Voluntaria del Embarazo (IVE), que fue presentado, desde el año 2007, en varias oportunidades ante la Cámara de Diputados de la Nación; finalmente, se despenalizó en el pasado mes de diciembre, y hoy se denomina Ley 27610 de $2021^{48}$.

Se debe considerar la diferencia entre despenalizar y legalizar. Lo primero implica "que el aborto no constituya más un delito que criminalice a las mujeres y a partir de esa decisión, pase a constituir el contenido de un derecho humano que obliga al Estado a garantizarlo"; mientras que legalizar "supone reconocer la interrupción voluntaria del embarazo de las mujeres como un derecho humano a la libertad de decidir sobre el cuerpo y a recibir por parte del Estado, como garante del derecho social a la salud pública, la cobertura de servicios para la realización de las prácticas correspondientes licitud e ilicitud"49.

- Como expresa Rita Segato "La lucha por la criminalización o descriminalización del aborto no es una lucha para que sea posible la práctica de este, pues la ley no ha demostrado su capacidad para controlarlo, sino por el acceso y por la inscripción de la narrativa jurídica de dos sujetos en pugna por obtener el reconocimiento en el contexto de la nación" y agrega "El autoritarismo de Estado se ejerce cuando se obliga a continuar con el emвarazo" $"$.

El movimiento político permitió, según la doctora Silvia Levín, modificar la praxis democrática en al menos tres $\operatorname{rasgos}^{49}$ :

1. Restablecer el valor de la democracia deliberativa como fuente de legitimidad de la ley, en la cual el debate público y la confrontación de posiciones $\mathrm{y}$ argumentaciones, a favor $\mathrm{y}$ en contra del aborto, prevalecieron como prácticas políticas institucionales y contribuyeron a organizar el centro de la toma de decisiones.

2. El conflicto inundó las calles, los hogares, las escuelas, las universidades, las plazas y las redes sociales en toda la Argentina, con manifestaciones masivas y políticas. La participación activa remarca un claro componente de influencia política para la toma de decisiones.

3. Las mujeres lograron ejercer un liderazgo transversal que neutralizó las diferencias sociales, ideológicas y religiosas. Mujeres adolescentes y jóvenes pusieron sus cuerpos en la calle como un grito vivo de la expresión de su derecho a la libertad de decidir.

El desafío feminista interpela al orden patriarcal (que, en alianza con el poder, la política y la teología, construyó e instrumentó el control y soberanía sobre los cuerpos de las mujeres durante siglos), para acelerar la instauración de un nuevo orden. Este debe estar inspirado en la libertad, se encamine al logro de un equilibrio de poder entre los géneros mediante la adquisición política de ciudadanía plena para las mujeres: el sexo en la esfera pública desvinculado de la reproducción y liberado a la facultad de las mujeres a decidir ejercerlo ${ }^{51}$. Así mismo, las desigualdades políticas de libertad, que arrastran otras tantas desigualdades, como las económicas y sociales, y que sostienen la institucionalidad democrática vigente: "Un embarazo no querido por una violación es idéntico a la violación" $"$.

No existen obstáculos constitucionales ni convencionales del sistema internacional de derechos humanos para la despenalización ni para la legalización del aborto, no obstante, solo cinco países de América Latina y el Caribe han logrado que una práctica histórica, que en condiciones de clandestinidad se 
transforma en muerte, dolor y oscurantismo, se inscriba hoy legalmente y reivindique la existencia y dignidad de las mujeres ${ }^{50}$ : "Con el aborto legal no habrá más ni menos abortos, habrá menos madres muertas".

\section{Enfoque ideal para prevención del aborto}

"Educación Sexual Integral para decidir, Programas integrales de Anticoncepción para no abortar y aborto legal para no morir".

En países donde desde hace varios años se involucró la educación sexual integral (ESI) en la enseñanza de los niños y adolescentes, con conceptos como valores, familia, respeto, convivencia, conocimiento del cuerpo, autonomía, resolución de problemas, toma asertiva de decisiones, autocuidado, etc., se ha logrado disminuir las tasas de embarazo adolescente. Sin embargo, no se desconoce que hay otros factores sociales, familiares, culturales y de servicios de salud que pueden influir en sus tasas de fecundidad ${ }^{52,53}$.

Para el cumplimiento de esta estrategia, desde el año 2010, la Organización de las Naciones Unidas para la Educación, la Ciencia y la Cultura (sigla en inglés UNESCO), en colaboración con el Fondo de Población de las Naciones Unidas (UNFPA), el Fondo de las Naciones Unidas para la Infancia (UNICEF), la OMS el Programa Conjunto de las Naciones Unidas sobre el VIH/sida (UNAIDS) desarrollaron un completo material pedagógico llamado "Orientaciones técnicas internacionales en educación sexual integral" ${ }^{2}$. Este documento ha sido actualizado en 2018 y busca que los educadores, servicios de salud y otras entidades involucradas con niños y adolescentes desarrollen e implementen programas de ESI para las escuelas y colegios. Los contenidos incluidos tienen una progresión apropiada para la edad y cuentan con la flexibilidad para incorporar elementos que forman parte de los contextos locales y comunitarios de cada población.

Sin embargo, la ESI es omitida por muchas instituciones o se desarrolla de forma no continua, y no aporta los claros y prácticos conceptos que el adolescente requiere $^{55}$. Existe evidencia ${ }^{16}$ de que la ESI, impartida desde los cinco años de edad, apropia al niño de un enfoque secuencial y adecuado para desarrollar de manera integral y responsable su comportamiento de vida, basado en el respeto consigo mismo y con los demás, en la autoprotección y en el correcto abordaje de deberes y derechos. De esta manera, se logra en el adolescente el desarrollo de habilidades que serán necesarias una vez decida el inicio de su vida sexual.
En este contexto, será más probable un desarrollo informado y responsable de su sexualidad, evitando los riesgos de embarazos no deseados, con alta posibilidad de finalizar en abortos inseguros ${ }^{5,55}$.

Otra estrategia importante en la prevención del aborto se encuentra en los servicios integrales de anticoncepción que deben aportar a la mujer y a su pareja un acompañamiento amigable, informado, continuo y científicamente correcto. Además, se debe lograr que la paciente tenga buena adherencia al método anticonceptivo y que su atención esté integrada a un programa completo de SSR. La OMS aporta documentos e información para que las competencias del personal de salud que trabaja en asesoría anticonceptiva se dé en el marco de derechos y se garantice la elección informada ${ }^{56}$. Adicionalmente, existen dos aplicaciones para teléfonos celulares con la información de los criterios de elegibilidad de la OMS que apoyan al profesional durante la asesoría y la toma de decisiones. Estos son: i-contraception y la rueda OMS (Wheel MEC-OMS) $)^{57}$.

La SSR debe ser el frente y el centro de la discusión en temas de desarrollo sostenible, acorde con el concepto de las organizaciones mundiales expertas en el tema ${ }^{58}$. Durante la celebración en julio de 2019 del $25 .^{\circ}$ aniversario internacional del Día de la Población Mundial y la Conferencia Internacional sobre Población y Desarrollo (ICDP) se ratificó que la planificación familiar es el epicentro de los temas demográficos y de $\mathrm{SSR}^{59}$ : "Un derecho básico para cada persona debería ser: contar con la habilidad de controlar su fertilidad, ejercer de forma planeada su deseo o no de tener hijos y de ser así, cuántos y cuándo...”

Es indudable que, al ser el aborto una situación que impacta la salud reproductiva de muchas mujeres y sus familias en el mundo, la educación sobre este tema merece un lugar relevante en los planes de estudio de todas las escuelas de medicina, e idealmente de otras áreas de la ciencias de la salud, de las humanidades y del derecho ${ }^{60}$.

En países donde la despenalización del aborto lleva muchos años, incluso décadas, como es el caso de Canadá, el personal médico cuenta con la instrucción necesaria para el abordaje y atención de las mujeres que solicitan el servicio. Esto, unido al advenimiento del Misoprostol para el manejo médico farmacológico del aborto temprano (idealmente antes de la semana 10), ya 
sea solo o en combinación con la Mifepristona, permite que la atención sea ágil, eficiente, digna y oportuna ${ }^{61}$.

Lo contrario ocurre en los países donde los prestadores del servicio de salud desconocen o incumplen los aspectos legislativos, humanos y técnicos para la atención del aborto. Además, existe escasa socialización del tema dentro de la población general, lo cual niega la posibilidad de una discusión amplia e integral y permite enfoques erróneos, bajo creencias morales y religiosas que llevan a establecer juicios y estigmatizaciones y a imponer barreras en la adecuada prestación del servicio y al acompañamiento médico, cuando se requiere ${ }^{62}$.

\section{Manejo médico del aborto}

Según datos del Centro para el Control y la Prevención de Enfermedades (de su nombre en inglés CDC), durante el año 2017, en Estados Unidos, 92,2\% de los abortos reportados en el año sucedieron antes de la semana $13 \mathrm{o}$ menos; el 77,7\% (3 de cada 4 mujeres) ocurrieron a las 9 semanas o menos, e incluso 4 de cada 10 mujeres lo realizó antes de la semana 6 (40,2\%). Estas cifras fueron relacionadas con la oportunidad del manejo del RU486 o Mifepristona, medicamento con efectiva acción antiprogestágena y aprobado en los Estados Unidos a partir del año 2000. Su uso fue inicialmente aprobado hasta la semana 7 y desde el año 2016 se aprobó para uso hasta la semana 10 por la FDA ${ }^{3}$. En Colombia, el medicamento fue introducido en el año 2017 por el Instituto Nacional de Vigilancia de Medicamentos y Alimentos (INVIMA) y aprobado para el manejo farmacológico de la IVE temprana ${ }^{63}$; sin embargo, la logística de su uso no es universal ni equitativa ya que su manejo es exclusivo de Profamilia.

En el año 2018, la OMS actualizó las dosis de misoprostol de acuerdo con la edad gestacional, la vía y el intervalo entre dosis, y subrayó la importancia de iniciar la anticoncepción posaborto oral o la inserción del DIU ${ }^{64}$. En octubre de 2020, el Colegio Americano de Obstetricia y Ginecología (ACOG) ratificó la efectividad de la combinación de los dos medicamentos y actualizó la guía del manejo médico farmacológico del aborto por debajo de la semana 10 con recomendaciones basadas en evidencia científica ${ }^{65}$. Adicionalmente, cabe recalcar que debido a la situación actual de la pandemia, la totalidad de la información y la asesoría para la IVE puede ser provista por telemedicina y los medicamentos se pueden enviar a las pacientes por correo ${ }^{61,65}$. Si el manejo farmacológico inicial no fuera exitoso y se evidenciaran signos físicos o ecográficos de aborto incompleto, se podría manejar una dosis adicional de misoprostol o una aspiración manual endouterina
(AMEU) o aspiración eléctrica, acorde con los recursos y entrenamiento del personal y con las circunstancias y preferencia de la paciente ${ }^{65}$. Es importante suministrar la información posaborto en relación con educación y asesoría en métodos anticonceptivos, siguiendo las recomendaciones de la OMS y la $\mathrm{CDC}^{66,67}$ y acorde con las características de cada paciente; en su mayoría, pueden ser iniciados desde el día siguiente del suministro de los medicamentos para el aborto.

\section{Telemedicina para atención del aborto}

En algunas regiones, la asesoría por telemedicina para el aborto médico temprano no es un tema exclusivo de la actual pandemia ${ }^{8}$ y lleva en uso más de una década. Esto muestra que no hay diferencias significativas en la prevalencia de eventos adversos cuando se atiende por telemedicina o cuando se da atención en persona a los casos de aborto por debajo de la semana 10 y que no se aumenta la posibilidad de eventos adversos graves como embarazo ectópico, requerimiento de cirugía o muerte $^{61,68}$. Sin embargo, en Colombia, a pesar de que la telemedicina está regulada desde el año $2010^{69} \mathrm{e}$ incluida en el plan de beneficios de salud, esta no ha tenido el desarrollo e implementación que debería.

\section{Conclusiones}

Argentina logró el pasado 29 de diciembre un fallo histórico respecto a la despenalización y legalización del aborto. Esta decisión fue apoyada con suficientes argumentos por diferentes asociaciones y agremiaciones de las ciencias de la salud que reúnen cerca de 7000 profesionales, entre ellas la Sociedad de Obstetricia y Ginecología de Buenos Aires (SOGIBA), la Asociación Médica Argentina de Anticoncepción, la Sociedad de Ginecología Infanto-Juvenil, la Federación Argentina de Medicina General y la Red de Profesionales de la salud por el Derecho a Decidir. Estas instituciones observan que la interrupción voluntaria del embarazo es un tema de salud pública y de respeto a los derechos sexuales y reproductivos de la mujer y, expresan que este avance constituye una herramienta visible que intenta generar un marco de igualdad de oportunidades para que toda persona que necesite tomar la difícil decisión de interrumpir la gestación (independientemente de cuál sea su causal, su situación socioeconómica y el lugar geográfico en que se encuentre) pueda tener acceso a un aborto en condiciones seguras, sin riesgo de vida. Esta debe ser gratuita, con un apoyo adecuado y un completo acompañamiento emocional, además, de la asesoría posparto con énfasis en anticoncepción. 
Es indudable que la información y experiencia sobre la ESI, impartida desde la infancia con continuidad, de forma progresiva, en currículo académico laico y sin diferenciación entre educación pública o privada, se convierte en la primera actividad con miras a la prevención de embarazos no deseados o no intencionales que son causa directa de la necesidad de solicitud de IVE por parte de las mujeres. Se hace necesario romper el estigma hacia los derechos sexuales y los reproductivos, de cara a garantizar el acceso a servicios de SSR con calidad y oportunidad, facilitando su ejercicio sin importar el género, la posición económica, la orientación sexual o la identidad de género, y dando mayor divulgación de los mismos. La OMS y demás organizaciones internacionales afines instan a los Estados a facilitar los espacios de diálogo que permitan el debate sano y constructivo en un marco de derechos con la participación de los diferentes sectores y en un contexto de bioética, priorizando programas y políticas en poblaciones más vulnerables para avanzar en la legalización del aborto en ALC, especialmente, en los países con leyes más restrictivas que prohíben de forma total el aborto.

Se deberán mejorar y agilizar los procesos en los sectores de salud y justicia para evitar la revictimización y la imposición de barreras de forma injustificada a mujeres que solicitan la IVE, principalmente, a quienes lo solicitan en segundo trimestre. Este hecho es frecuente en niñas y adolescentes que son víctimas de violación y que descubren su embarazo en periodos avanzados o porque tardíamente tienen la capacidad de denunciarlo. También incluye aquellas situaciones que inician como embarazos deseados y posteriormente se tornan en indeseados o inoportunos, ya sea a causa de malformaciones fetales o riesgos graves para la salud de la madre. Ello somete a las mujeres a sufrimientos innecesarios, sobre todo cuando existen técnicas seguras, cuya disponibilidad podría estar al alcance de quienes lo requieran.

Por último, se sugiere reconocer el aporte de la telemedicina para el acceso a un aborto legal y seguro en medio del confinamiento y del distanciamiento social. Este servicio de salud es una oportunidad para que las mujeres de diferentes regiones puedan acceder a la IVE con la asesoría de personal especializado, contribuyendo, así, a la autonomía de las mujeres en este momento de crisis.

\section{Referencias}

1. Bearak J, Popinchalk A, Ganatra B, Moller AB, Tunçalp Ö, Beavin $C$, et al. Unintended pregnancy and abortion by income, region, and the legal status of abortion: estimates from a comprehensive model for 1990-2019. Lancet Glob Heal. 2020; 8(9): e1152-1161.

2. Ministerio de Salud y Protección Social y Fondo de Población de las Naciones Unidas (UNFPA). Determinantes del aborto inseguro y barreras de acceso para la atención de la interrupción voluntaria del embarazo en mujeres colombianas. 1. ${ }^{\mathrm{a}}$ ed. Bogotá: Ministerio de Salud y Protección Social; 2014.

3. O'Bannon RK. CDC Updates Abortion figures show short term increase in 2018 but long term decline. National Right to Life News; 2020. https:// www.nationalrighttolifenews.org/2020/12/cdcupdates-abortion-figures-show-short-term-increasein-2018-but-long-term-decline/

4. Malta M, Wells S, LeGrand S, Seixas M, Baptista A, Furtado Passos da Silva CM, et al. Abortion in Brazil: the case for women's rights, lives, and choices. The Lancet. 2019; 4(11): e552.

5. Zulu JM, Haaland MES. Situating the Mexico City Policy: what shapes contraceptive access and abortion? Lancet Glob Heal. 2019; 7(8): e984-985.

6. La Mesa por la Vida y la Salud de las Mujeres. Barreras de acceso a la Interrupción Voluntaria del Embarazo en el Contexto de la pandemia por COVID-19. 2020.

7. Ministerio de Salud y Protección Social y Fondo de Población de las Naciones Unidas (UNFPA). Millones de mujeres sufrirán embarazos no deseados durante la pandemia de coronavirus. 2020. https:// news.un.org/es/story/2020/04/1473572

8. UNFPA. Preparación y Respuesta a la Enfermedad del Coronavirus (COVID-19) Resumen Técnico Provisional del UNFPA; 2020. p. 1-5. https:// www.unfpa.org/sites/default/files/resource-pdf/ COVID19-TechBrief-SSR-23Mar20.pdf

9. Interagency Working Group on Reproductive Health in Crisis. Orientación programática para la salud sexual y reproductiva en contextos humanitarios y frágiles durante la pandemia del COVID-19. cdn.iawg.rygn.io. 2020. https://reliefweb.int/ sites/reliefweb.int/files/resources/Orientación programática para la salud sexual y reproductiva en contextos humanitarios y frágiles durante la pandemia de la COVID-19.pdf 
10. Ministerio de Salud y Protección Social de Colombia. Resolución 536 de 2020. insalud.gov.co. 2020. https://www.minsalud.gov.co/Normatividad_ Nuevo/Resolución No. 536 de 2020.pdf

11. Ministerio de Salud y Protección Social. Resolución 3280 de 2018: Ruta de Atención en Salud Materno Perinatal, Modificada por la Resolución 276 de 2019. Colombia; 2018.

12. Corte Constitucional de Colombia. Sentencia C-355/06. Colombia; 2006.

13. Ministerio de Salud y Protección Social y Fondo de Población de las Naciones Unidas (UNFPA). Prevención del Aborto Inseguro en Colombia Protocolo para el Sector Salud. 1a ed. Bogotá: Ministerio de Salud y Protección Social; 2014; $1-114 \mathrm{p}$.

14. Organización mundial de la salud. Prevención del aborto peligroso. who.int; 2020. https://tinyurl. com/yyrgec $4 \mathrm{r}$

15. World Health Organization. Safe abortion: technical and policy guidance for health systems. Vol. 2, World Health Organization. World Health Organization; 2012. $64 \mathrm{p}$.

16. Ministerio de Salud y Protección Social y Fondo de Población de las Naciones Unidas (UNFPA). Orientación y Asesoría para la Interrupción Voluntaria del Embarazo (IVE). Documento Técnico para Prestadores de Servicios de Salud. $1^{\text {a }}$ ed. Bogotá: Ministerio de Salud y Protección Social; 2014.

17. Ministerio de Salud y Protección Social y Fondo de Población de las Naciones Unidas (UNFPA). Atención integral de la interrupción voluntaria del embarazo (IVE) en el primer nivel de complejidad. Documento técnico para prestadores de servicios de salud. $1^{\text {a }}$ ed. Bogotá: Ministerio de Salud y Protección Social; 2014.

18. World Health Organization. Health worker roles in providing safe Abortion Care and Post-Abortion Contraception. World Health Organization; 2015.

19. Ministerio de Salud y Protección Social y Fondo de Población de las Naciones Unidas (UNFPA). Guía de capacitación para atención en salud de la interrupción voluntaria del embarazo. Bogotá: Ministerio de Salud y Protección Social; 2014.

20. Haití. Código penal de Haití. Art 262. 1835 p. 634.

21. Congreso Nacional de República Dominicana. Codigo Penal de la Republica Dominicana. Art. $317 ; 1884$.

22. La asamblea legislativa de la república del Salvador. Código Penal El Salavador, Artículo. 133-137; 1997.
23. El congreso nacional. Codigo Penal de Honduras. Art. 126-132; 1983.

24. El congreso nacional. Código penal de Honduras Decreto 130-2017, Art. 196. 2019.

25. Asamblea Nacional de Nicaragua. Proyecto de Ley No. 641, Código penal; Capítulo II. Art.143-145; 2007.

26. Diario Oficial de la Federación. Código Penal Federal Art. 329-334. Diario Oficial de la Federación; 2020 p. 320 .

27. Congreso de la República de Guatemala. Código Penal de guatemala, Decreto №. 17-73, Título VII, Capítulo III, Artículos 133-140; 1973.

28. PGN. Texto Único Del Código Penal De La República De Panamá, Capítulo 1, Sección 3; Aborto provocado. Art. 141-144; 2015.

29. La comisión legislativa. Código Penal de Venezuela, Título VIII, Capítulo IV; Del aborto provocado. Art. 432-436; 2000.

30. Presidente de la república. Decreto-Lei № 2.848 , de 07.12.1940, alterado pela Lei № 9.777 em 26/12/98. Codigo Penal Brasil. Art 124-128; 1940.

31. Corte suprema de Justicia. Codigo penal de la republica del Paraguay Art. 349-353; 2001.

32. Ministerio de Salud (MINSAL). Acompañamiento y atención integral a la mujer que se encuentra en alguna de las tres causales que regula la ley 21.030; 2018.

33. La asamblea legislativa de la República de Costa Rica. Código Penal Ley No 4573. Art. 118-122 y $381 ; 1970$.

34. Reino unido. Abortion Act 1967 Chapter 87; 1967.

35. Ministerio de Justicia Derechos Humanos y Cultos. Código orgánico integral penal de ecuador, Art. 147 - 150; 2000.

36. Poder Ejecutivo del Perú. Código Penal Capítulo II, Art. 114-120; 1991.

37. Bolivia. Código Penal de Bolivia, Título VIII, Capítulo II, Artículos 263 - 269. 1972.

38. Laws Substantive Commissioner. Customs and Excise Duties Act Chapter 48 Edition 2011 Showing the Substantive Laws As At. Title IX, Art. 111, 112, $127 ; 2005$.

39. CPCM. Código Penal para la Ciudad de México. Artículos 144-150; 2002.

40. BBC. Cuba: cuando el aborto es una alternativa al método anticonceptivo; 2011. https://www.bbc.com/ mundo/noticias/2011/03/110119_cuba_aborto_ salud_lh\#: : text=Aunque en Cuba no existe,para optar por esa alternativa

41. María D, Benítez E. La trayectoria del aborto seguro en Cuba: evitar mejor que abortar. Rev Noved Población. 2014; 10(20): 87-104. 
42. Cuba. Código penal de cuba Capítulo IV Art. 267271. 1987.

43. Code de la santé publicque. Code de la santé publique (Code of Public Health) Chapitre II: Interruption pratiquée avant la fin de la douzième semaine de grossesse. (Articles L2212-1 à L2212-11). 1953.

44. Argentina. Ley interrupción voluntaria del embarazo 30 diciembre 2020.

45. Uruguay. Sentencia No 586/2015. J Res. 2015; 44(12): 243-254.

46. France24. Haití marcha hacia la despenalización del aborto. france24.com. 2020. https://www. france24.com/es/20200717-haití-marcha-hacia-ladespenalización-del-aborto

47. Aborto legal. La Campaña Nacional por el Derecho al Aborto legal, Seguro y Gratuito. abortolegal.com. ar. 2020. http://www.abortolegal.com.ar/about/

48. Boletín oficial de la república de Argentina. Acceso a la interrupción voluntaria del embarazo. 2020. https:/www.boletinoficial.gob.ar/detalleAviso/ primera/239807/20210115

49. Levín S. Sexual and reproductive health without freedom?: The conflict over abortion in Argentina. Salud Colect. 2018; 14(3): 377-389.

50. Perugino S. La despenalización del aborto en Argentina. Disputas en torno a la narrativa legal. Con X. 2016; 2: e011. https://perio.unlp.edu.ar/ojs/ index.php/conequis/article/view/CXe011

51. Parlamentario Ricardo Gil Lavedra: "La maternidad es una opción, no una obligación" 2018. https://www. parlamentario.com/2018/05/31/ricardo-gil-lavedrala-maternidad-es-una-opcion-no-una-obligacion/

52. Chandra-Mouli V, Camacho AV, Michaud PA. WHO guidelines on preventing early pregnancy and poor reproductive outcomes among adolescents in developing countries. J Adolesc Heal. 2013; 52(5): 517-522.

53. Lindberg LD, Maddow-Zimet I. Consequences of sex education on teen and young adult sexual behaviors and outcomes. J Adolesc Heal. 2012; 51(4): 332-338.

54. UNESCO. International technical guidance on sexuality education: an evidence-informed approach- es.unesco.org. 2018. https://unesdoc. unesco.org/ark:/48223/pf0000265335

55. Vanwesenbeeck I, Westeneng J, de Boer T, Reinders J, van Zorge R. Lessons learned from a decade implementing Comprehensive Sexuality Education in resource poor settings: The World Starts With Me. Sex Educ. 2016; 16(5): 471-486.
56. Organización Mundial de la Salud. Recomendaciones sobre prácticas seleccionadas para el uso de anticonceptivos. Organización Mundial de la Salud; 2018.

57. Organización Mundial de la Salud. Rueda con los criterios medicos de elegibilidad para el uso de anticonceptivos. Organización Mundial de la Salud; 2016.

58. Fondo de Población de las Naciones Unidas (UNFPA). Planificación Familiar: Clave para el Desarrollo Sostenible. 2017. https://lac.unfpa. org/es/news/planificación-familiar-clave-para-eldesarrollo-sostenible-1

59. The Lancet Global Health. SRHR: unmet needs and unfinished agendas. Lancet Glob Heal. 2019; 7(8): e979.

60. Heger J, Roth J KS. What Are We Learning? An Update on Abortion Education in Medical Schools [29G]. Obstet Gynecol. 2020; 78s.

61. Bancsi A GK. Update on medical abortion. Canadian family physician. 2018. https://www.caps-cpca. ubc.ca/AnnokiUploadAuth.php/e/e0/Canadian_ Resource_1__Medical_Abortion_Prescriber_ Checklist_2018-07-11.pdf

62. Braga W, Castro A, Maria S, Leite DL, Cunha T. Percepción de profesionales de la salud sobre el aborto legal. Rev Bioét. 2015; 23(2): 394-406.

63. Ministerio de Salud y Protección Social e Instituto Nacional de Vigilancia de Medicamentos y Alimentos INVIMA. Registro sanitario Mifepristona. 2016.

64. World Health Organization. Medical management of abortion. Geneva: WHO; 2018. https://tinyurl. com/y31zpjuh

65. Creinin M, Grossman D. Medication Abortion Up to 70 Days of Gestation. ACOG PRACTICE BULLETIN. Clinical Management Guidelines for ObstetricianGynecologists. 2020; 136(143): 855-858.

66. Organización Mundial de la Salud. Medical eligibility criteria for contraceptive use. OMS. 2015.

67. Centers for Disease Control and Prevention. Criterios Médicos de Elegibilidad para el Uso de Anticonceptivos. CDC. 2017.

68. Grossman D, Grindlay K. Safety of medical abortion provided through telemedicine compared with in person. Obstet Gynecol. 2017; 130(4): 778-782.

69. Congreso de la Republica de Colombia. Ley 1419 de 2010. Congreso de Colombia 2010. 